\title{
Submillimeter Surveys at High Redshift
}

\author{
Amy J. Barger \\ Institute for Astronomy, University of Hawaii, 2680 Woodlawn Dr., \\ Honolulu, HI 96822
}

\begin{abstract}
Deep submillimeter (submm) surveys offer an unobscured view of dust-enshrouded star formation or AGN activity at high redshifts. SCUBA observations above $2 \mathrm{mJy}$ have resolved $20-30 \%$ of the far-infrared (FIR) background into discrete sources and have revealed the existence of a distant population of galaxies with properties similar to those of local ultraluminous infrared galaxies. A large fraction of the submm sources have extremely faint optical/near-infrared (NIR) counterparts and hence are inaccessible to optical spectroscopy. Millimetric redshift estimation places the submm population at $z=1$ to 3 . While the cumulative surface density of the submm sources is low, they are so luminous that if powered mainly by star formation, they dominate the high redshift star formation history. Recent combined SCUBA submm and Chandra hard X-ray studies suggest that the majority of the submm sources are star formers with only a small admixture of obscured AGN.
\end{abstract}

\section{Introduction}

The cumulative emission from all objects lying beyond the Galaxy, the extragalactic background light (EBL), provides important constraints on the integrated star formation history of the Universe. The recent measurements of the EBL at FIR and submm wavelengths using data from the FIRAS and DIRBE experiments on the COBE satellite (e.g., Puget et al. 1996; Fixsen et al. 1998) indicate that the total emission from star formation and AGN activity that is absorbed by dust and reradiated into the FIR/submm is comparable to the unobscured emission seen in the optical. This suggests that obscured star formation may be responsible for a large fraction of the stars and metals seen in the local Universe.

Reradiation of light by dust into the FIR produces a thermal emission peak at $\lambda \sim 100 \mu \mathrm{m}$ that is redshifted into the submm for galaxies at $z>1$. Submm observations are unique in having a strong negative $\mathrm{K}$-correction that compensates for cosmological dimming beyond $z \simeq 1$, thereby making dusty galaxies almost as easy to detect at $z \simeq 10$ as at $z \simeq 1$. The Submillimeter Common User Bolometer Array (SCUBA; Holland et al. 1999) on the James Clerk Maxwell 15 $\mathrm{m}$ telescope on Mauna Kea provides the sensitivity and area coverage to image directly the sources producing the submm EBL. SCUBA surveys have uncovered numerous sources with properties similar to those expected for the distant counterparts to the most luminous, merging systems observed locally, the ultra- 
luminous infrared galaxies (ULIGs; Sanders \& Mirabel 1996). If the majority of the submm emission in these systems comes from dust-obscured star formation, then their inferred star formation rates are spectacular, on the order of several hundred solar masses per year.

\section{Submillimeter Counterparts and the Radio Advantage}

Identifying the optical/NIR counterparts to the submm sources is difficult due to the uncertainty in the SCUBA positions and the intrinsic faintness of the sources. Barger et al. (1999) presented a spectroscopic survey of possible optical counterparts to a flux-limited sample of galaxies selected from the $850 \mu \mathrm{m}$ survey of massive lensing clusters by Smail, Ivison, \& Blain (1997). Candidate optical counterparts in the SCUBA error-boxes were identified using moderately deep ground-based and $H S T$ exposures $(I \sim 23.5$ and $I \sim 26$, respectively). Onequarter of the sources could be reliably identified, and those had redshifts in the range $z \sim 1$ to 3 . A lower limit of $20 \%$ of the full sample showed signs of AGN activity. However, for the majority of the submm sources there were either no optical counterparts or the optical associations were not secure.

High resolution radio continuum maps with subarcsecond positional accuracy and resolution offer new opportunities for locating submm sources and determining their physical properties. About $20 \%$ of the sources in an ultradeep radio map of the Hubble Deep Field region at $1.4 \mathrm{GHz}$ (Richards 2000) are undetected in ground-based optical imaging to $I \sim 25$, and this population has been shown to contain the bright submm source population (Barger, Cowie, \& Richards 2000). An important corollary is that a large fraction of the sources in submm surveys have extremely faint optical counterparts and hence are inaccessible to optical spectroscopy. Redshift estimates made from the ratios of the submm fluxes to the radio fluxes (Carilli \& Yun 2000) place the bright submm sources at $z \sim 1$ to 3 where they form the high redshift tail of the faint radio population.

\section{Towards a Star Formation History}

Observations of high redshift sources are often interpreted using the comoving volume-averaged history of star formation diagrams introduced by Madau et al. (1996). Measurements are typically based on the rest-frame ultraviolet (UV) luminosity function, whose origin is assumed to be young stellar populations. However, dust plays an important role in the high redshift Universe. Recent estimates suggest that dust obscuration of high redshift optical sources result in upward corrections by factors of 3 to 5 (e.g., Pettini et al. 1998; Meurer, Heckman, \& Calzetti 1999) in the star formation rate density, but these corrections are rather uncertain. Moreover, many objects may be so extinguished that ultimately only submm studies can reliably determine how much light is hidden, and, in particular, whether the most rapid and obscured massive star formers are completely missed in optical surveys.

Any attempt at tracing the dust-obscured star formation history of the Universe also requires knowledge of the extent of the contribution that AGN make to powering the submm sources. X-ray surveys provide the most direct and un- 
biased probe of massive black hole accretion activity throughout the Universe, particularly in the hard X-ray band above $2 \mathrm{keV}$ where most of the energy density of the extragalactic X-ray background (XRB) resides. Early observations with the Chandra satellite have resolved more than $70 \%$ of the $2-8 \mathrm{keV} \mathrm{XRB}$ into point sources (Mushotzky et al. 2000; Garmire et al., in preparation). Unexpectedly, approximately half of the hard XRB emission is from relatively local luminous early-type galaxies in the redshift range $z=0$ to 1 (Barger et al. 2001). The X-rays arise from the cores of these galaxies, presumably as a result of AGN activity, but most of the galaxies show no clear AGN signatures in their optical spectra. Most of the remainder of the XRB emission is produced by much fainter optical sources $(I>23.5)$ whose colors are consistent with evolved early galaxies at $z>1.5$. If the hard X-ray sources are highly absorbed, then the rest-frame soft X-ray through NIR radiation will be reprocessed by dust and gas and the energy will appear in the FIR.

\section{Hard X-ray Properties of a Submillimeter Selected Sample}

The results of recent searches for Chandra hard X-ray counterparts to submm sources have been mixed. In a study of two clusters by Fabian et al. (2000), only a marginal source was seen in both the X-ray $(2.8 \sigma)$ and submm $(2 \sigma)$ datasets. Likewise, none of the submm sources in the ultradeep Hubble Deep Field SCUBA map of Hughes et al. (1998) was detected in the $2-8 \mathrm{keV}$ band by Hornschemeier et al. (2000). In contrast, two of the submm sources in the A370 lensed field of Smail, Ivison, \& Blain (1997) were detected in the $2-10 \mathrm{keV}$ band by Bautz et al. (2000). These two sources had previously been identified spectroscopically as AGN (Ivison et al. 1998; Barger et al. 1999). The above mixed results probably reflect the fact that the $850 \mu \mathrm{m}$ flux limits obtainable with SCUBA are quite close to the expected fluxes from obscured AGN.

Barger et al. (2001) compared wide-area and ultradeep submm maps (Barger, Cowie, \& Sanders 1999; Barger et al. 1998) with the SSA13 deep Chandra X-ray map of Mushotzky et al. (2000). The $2-10 \mathrm{keV}$ fluxes for each of the twelve submm sources detected in the high quality X-ray region were measured in $10^{\prime \prime}$ diameter apertures. Only one submm source was found to be a strong hard Xray emitter. The submm source with the second strongest hard X-ray flux is not a significant hard X-ray source but is a known soft X-ray emitter (Mushotzky et al. 2000).

The ratio of the total hard X-ray flux in the sample to the total submm flux is $1.4 \pm 0.5 \times 10^{-16} \mathrm{erg} \mathrm{cm}^{-2} \mathrm{~s}^{-1} \mathrm{mJy}^{-1}$. This ratio can be used to estimate the fraction of the hard XRB that arises from submm sources in this flux range. The EBL of the $2-10 \mathrm{mJy}$ source population is $9.3 \times 10^{3} \mathrm{mJy} \mathrm{deg}^{-2}$ (Barger, Cowie, \& Sanders 1999), which would contribute $1.3 \times 10^{-12} \mathrm{erg} \mathrm{cm}^{-2} \mathrm{~s}^{-1} \mathrm{deg}^{-2}$ in the $2-10 \mathrm{keV}$ band or $6 \%$ of the hard XRB, if the Vecchi et al. (1999) value of $2.3 \times 10^{-11} \mathrm{erg} \mathrm{cm}^{-2} \mathrm{~s}^{-1} \mathrm{deg}^{-2}$ is adopted.

However, nearly all of the X-ray signal from the submm sample is coming from one source. If this single source is removed, the total hard X-ray to total submm flux ratio drops to $0.6 \pm 0.5 \times 10^{-16} \mathrm{erg} \mathrm{cm}^{-2} \mathrm{~s}^{-1} \mathrm{mJy}^{-1}$. If the submm sources are assumed to lie at $z=2$ (consistent with the $z=1-3$ spectroscopic and millimetric redshift range for submm sources), then the $1 \sigma$ lower limit on 
the ratio of the FIR luminosity to the hard X-ray luminosity is approximately 1100. This lower limit is above the values for obscured AGN $(\sim 10-100)$ and approaching that of Arp $220\left(3.4 \times 10^{4}\right)$. It therefore appears that most of the submm sources, at least above $2 \mathrm{mJy}$, are star formers with a small admixture of obscured AGN.

\section{Acknowledgments}

I thank David Wilner for inviting me to this stimulating conference. I am very grateful for an IAU Travel Grant and for a travel award made possible through a grant to the AAS from the NSF and a grant from NASA. I thank L. Cowie, R. Mushotzky, E. Richards, and K. Arnaud for collaborations on this work. The work presented here was supported by NASA through Hubble Fellowship grant HF-01117.01-A awarded by the Space Telescope Science Institute, which is operated by the Association of Universities for Research in Astronomy, Inc., for NASA under contract NAS 5-26555.

\section{References}

Barger, A.J., et al. 1998, Nature, 394, 248

Barger, A.J., Cowie, L.L., Sanders, D.B. 1999, ApJ, 518, L5

Barger, A.J., et al. 1999, AJ, 117, 2656

Barger, A.J., Cowie, L.L., Richards, E.A. 2000, AJ, 119, 2092

Barger, A.J., Cowie, L.L., Mushotzky, R.F., Richards, E.A. 2001, AJ, submitted, [astro-ph/0007175]

Bautz, M.W., et al. 2000, ApJ, in press, [astro-ph/0008050]

Carilli, C.L., Yun, M.S. 2000, ApJ, 530, 618

Fabian, A.C., et al. 2000, MNRAS, 315, L8

Fixsen, D.J., et al. 1998, ApJ, 508, 123

Holland, W.S., et al. 1999, MNRAS, 303, 659

Hornschemeier, A.E., et al. 2000, ApJ, in press, [astro-ph/0004260]

Hughes, D.H., et al. 1998, Nature, 394, 241

Ivison, R., et al. 1998, MNRAS, 298, 583

Madau, P., et al. 1996, MNRAS, 283, 1388

Meurer, G.R., Heckman, T.M., Calzetti, D. 1999, ApJ, 521, 64

Mushotzky, R.F., Cowie, L.L., Barger, A.J., Arnaud, K.A. 2000, Nature, 404, 459

Pettini, M., et al. 1998, ApJ, 508, 539

Puget, J.-L., et al. 1996, A\&A, 308, L5

Richards, E.A. 2000, ApJ, 533, 611

Sanders, D.B., Mirabel, I.F. 1996, ARA\&A, 34, 749

Smail, I., Ivison, R.J., Blain, A.W. 1997, ApJ, 490, L5

Vecchi, A., et al. 1999, A\&A, 349, L73 